SLAC-PUB-95-7041

IASSNS-HEP-95/114

NSF-ITP-95-149

hep-th/9603158

\title{
Dynamical Supersymmetry Breaking on Quantum Moduli Spaces
}

\author{
Kenneth Intriligator \\ Institute for Advanced Study \\ Princeton, NJ 08540 \\ Scott Thomas \\ Stanford Linear Accelerator Center \\ Stanford University \\ Stanford, CA 94309 \\ and \\ Institute for Theoretical Physics \\ University of California \\ Santa Barbara, CA 93106
}

Supersymmetry breaking by the quantum deformation of a classical moduli space is considered. A simple, non-chiral, renormalizable model is presented to illustrate this mechanism. The well known, chiral, $S U(3) \times S U(2)$ model and its generalizations are shown to break supersymmetry by this mechanism in the limit $\Lambda_{2} \gg \Lambda_{3}$. Other supersymmetry breaking models, with classical flat directions that are only lifted quantum mechanically, are presented. Finally, by integrating in vector matter, the strongly coupled region of chiral models with a dynamically generated superpotential is shown to be continuously connected to a weakly coupled description in terms of confined degrees of freedom, with supersymmetry broken at tree level. 


\section{Introduction}

Non-perturbative gauge dynamics can lead to supersymmetry breaking in theories in which supersymmetry is unbroken at tree level. The "classic" models of dynamical supersymmetry breaking [1,2] all relied on dynamically generated superpotentials. This leads to a potential which lifts the classical moduli space and drives scalar fields to large expectation values. Additional tree level interactions can give a potential which rises at large expectation values, thereby leading to a stable ground state. In certain circumstances the auxiliary components and potential do not vanish in such a ground state, and supersymmetry is spontaneously broken. Recent advances led by Seiberg [3, 4], however, have shown that many supersymmetric theories have other types of non-perturbative dynamics, which lead to exactly degenerate quantum moduli spaces of vacua rather than dynamically generated superpotentials. In some cases the quantum moduli space is smooth and the low energy theory consists only of the massless moduli everywhere on the space. In other cases, there are additional non-perturbative light fields at various points on the moduli space. See [5] for a recent review and references. The quantum dynamics of theories with moduli spaces of vacua could in principle lead to new mechanisms for supersymmetry breaking, even though the dynamical superpotential exactly vanishes on the classical moduli space. The possibility of breaking supersymmetry as the result of a smooth quantum moduli space (with confinement at the origin) was considered in a simple model in Ref. [6]. Here we consider models in which supersymmetry is broken as a result of the quantum deformation of a classical moduli space constraint.

The mechanism of supersymmetry breaking by the quantum deformation of a moduli space relies on the observation that the patterns of breakings for global or gauge symmetries

on a quantum moduli space may differ from those on the classical moduli space. For example, since moduli typically transform under global symmetries, there is a point on the classical moduli space at which all the fields have zero expectation value, and the global symmetries are unbroken. However, in the quantum theory some of the global symmetries can remain broken everywhere on the moduli space [3]. Points which are part of the classical moduli space can therefore be removed by the quantum deformation. If tree level interactions give vanishing potential and auxiliary components only at points on the classical moduli space which are not part of the quantum deformed moduli space, supersymmetry is broken in the quantum theory.

The superpotential and spectrum of light fields on moduli spaces of vacua can often be 
determined exactly. However, in theories in which supersymmetry is broken, a quantitative discussion of the ground state and excited spectrum also requires knowledge of the Kahler potential. This can only be approximately determined in certain limits. One such limit is when the expectation values in the ground state are much larger than all dynamical scales. The gauge dynamics are then weak, and the Kahler potential is approximately the classical one for the underlying fields projected onto the classical moduli space. As we illustrate in several examples, another limit in which the Kahler potential can be approximately determined is near points of enhanced symmetry on the quantum moduli space of vacua. In this limit it is often possible to use 't Hooft anomaly matching conditions to find the correct degrees of freedom. For small expectation values away from such points the Kahler potential should be approximately canonical in these degrees of freedom, up to small corrections suppressed by the dynamical scale. This information about the Kahler potential played an important role in [6] and will be exploited here as well. It allows an analysis in regions which may have naively been thought to be strongly coupled and inaccessible.

In the next section we present the simplest example of supersymmetry breaking via the quantum deformation of a classical moduli space constraint. Taking the Yukawa coupling in the model to be small allows an analysis in various limits by perturbing about the quantum moduli space. This model also provides an example of a renormalizable model in which singlet fields participate directly in the supersymmetry breaking. In the third section, we discuss the well known $S U(3) \times S U(2)$ model of Affleck, Dine, and Seiberg [2]. We show that supersymmetry breaking in the limit $\Lambda_{2} \gg \Lambda_{3}$ is associated with the quantum deformation of a classical moduli space constraint due to the $S U(2)$ dynamics, rather than a dynamically generated superpotential arising from the $S U(3)$ dynamics. In the fourth section we present some generalizations of this model and determine the scaling of the vacuum energy in various limits. In the fifth section, we present models which break supersymmetry even though they have classical flat directions which are not lifted by the tree level superpotential. These directions are only lifted by the tree level superpotential in the presence of quantum effects. In section six we discuss the addition of vector-like matter to theories which break supersymmetry by a dynamically generated superpotential. Taking the mass of the vector-like matter much less than the dynamical scales allows these theories to be connected to a weakly coupled description in terms of confined degrees of freedom. Demonstrating supersymmetry breaking in the theory with extra matter gives confidence 
that in the original theory there are not additional branches at strong coupling along which supersymmetry is restored. These models also provide examples of supersymmetry breaking based on confinement. Finally, in section seven we present our conclusions and discuss possible applications of the simple model of section two. We also comment on general features of non-chiral models which break supersymmetry, and point out how the Witten index can vanish in such models.

\section{2. $S U(2)$ Quantum Moduli Space with Singlets}

The simplest example of a moduli space with a quantum deformed constraint is $S U(2)$ with four doublet matter fields $Q_{i}, i=1, \ldots, 4$. The classical moduli space is parameterized by the gauge invariants $M_{i j}=Q_{i} Q_{j}$ subject to the constraint Pf $M \equiv \epsilon^{i j k l} M_{i j} M_{k l}=0$. Quantum mechanically, the constraint is modified to $\operatorname{Pf} M=\Lambda_{2}^{4}$ [3]. While the point $M_{i j}=0$ is part of the classical moduli space, it does not lie on the quantum moduli space.

Supersymmetry would be broken if the quantum modification of a moduli space were incompatible with a stationary superpotential, $W_{\phi} \neq 0$, where $\phi$ is any field. For example, in the $S U(2)$ case mentioned above, supersymmetry would be broken if there were $F$ terms which only vanished for $M_{i j}=0$. A simple realization of this is to add to the $S U(2)$ model given above, six singlet fields $S^{i j}=-S^{j i}$, where $i, j=1, \ldots, 4$, with couplings 《7]

$$
W_{\text {tree }}=\lambda S^{i j} Q_{i} Q_{j}=\lambda S^{i j} M_{i j}
$$

This superpotential leaves invariant an $S U(4)_{F}$ flavor symmetry under which $Q_{i}$ transform as 4 , and $S^{i j}$ transform as $\mathbf{6}$. There is also an anomaly free $U(1)_{R}$ symmetry under which $R(Q)=0$ and $R(S)=2$. Any mass terms $m Q Q$ can be absorbed in a shift of the $S^{i j}$. The form of the couplings (2.1) can be enforced by discrete symmetries, or by weakly gauging certain subgroups of the flavor symmetry. The absence of any higher order terms in $S$ which respect the $S U(4)_{F}$ flavor symmetry, such as $(\mathrm{Pf} S)^{k}$, can be enforced by gauging a discrete subgroup of $U(1)_{R}$. Because the fields $S^{i j}$ do not appear alone to any power in the superpotential, they are similar to the moduli of string theory which parameterize flat directions of the perturbative potential. Classically, there is a moduli space of supersymmetric vacua with $M_{i j}=0$ and $S^{i j}$ arbitrary. Quantum mechanically, the $S^{i j}$ equations of motion, $\lambda M_{i j}=0$, are incompatible with the quantum constraint $\operatorname{Pf} M=$

$\Lambda_{2}^{4}$. The classical moduli space of supersymmetric vacua is completely lifted for $\lambda \neq 0$ as 
a result of the quantum modification of the $S U(2)$ moduli space, and supersymmetry is broken.

The modification of the moduli space can be realized in the superpotential with a Lagrange multiplier to enforce the quantum constraint. The full superpotential can then be written as

$$
W=\lambda S M+\mathcal{A}\left(\operatorname{Pf} M-\Lambda_{2}^{4}\right)
$$

where $\mathcal{A}$ is a Lagrange multiplier field. For $\lambda \ll 1$ the supersymmetry breaking ground state lies close in field space to the $S U(2)$ quantum moduli space, so the model may be analyzed by perturbing about this space. This amounts to enforcing the $\mathcal{A}$ equation of motion and analyzing the resulting potential in terms of the remaining light degrees of freedom. This is physically reasonable, as the modes which take the system away from the quantum moduli space are expected to have mass of at least $\mathcal{O}\left(\Lambda_{2}\right)$. For $\lambda \neq 0$ the heavy and light states of course mix, as shown below explicitly. However, for $\lambda \ll 1$ the mixing is small and does not affect the leading behavior of physical quantities such as the vacuum energy. For $\lambda \sim 1$ supersymmetry is still broken but can not be described in any quantitative way in terms of the $S^{i j}$ and $M_{i j}$ degrees of freedom.

The description of supersymmetry breaking in the $\lambda \ll 1$ limit depends on the values $\lambda S^{i j}$. For $\lambda S^{i j} \ll \Lambda_{2}$, the mesons $M_{i j}$ are light and should not be integrated out. For $\lambda=0$, the quantum constraint breaks the $S U(4)_{F}$ flavor symmetry at a generic point to $S U(2)_{F} \times S U(2)_{F}$, and at an enhanced symmetry point to $S P(2)_{F} \sim S O(5)_{F}$ [3]. Enhanced symmetry points are always extrema of the full potential, so are good candidates for minima. At the enhanced symmetry point the $M_{i j}$ have the form

$$
M_{0}= \pm\left(\begin{array}{ll}
i \sigma_{2} & \\
& i \sigma_{2}
\end{array}\right) \Lambda_{2}^{2} .
$$

The fluctuations of $M^{i j}$ away from (2.3) subject to the quantum constraint $\operatorname{Pf} M=\Lambda_{2}^{4}$ are fields $M_{5}$ in the $\mathbf{5}$ of $S O(5)_{F}$. The fields $\widehat{M}_{5}=M_{5} / \Lambda_{2}$ comprise the massless spectrum near the vacuum (2.3), as evidenced by the 't Hooft anomaly matching for the unbroken flavor symmetry [3]. Because these fields are the relevant degrees of freedom at (2.3), for small expectation values they have canonical Kahler potential up to small corrections, $K=\widehat{M}_{5}^{\dagger} \widehat{M}_{5} g\left(t=\widehat{M}_{5}^{\dagger} \widehat{M}_{5} /\left|\Lambda_{2}\right|^{2}\right)$ where $g(0)=1$. Under the $S O(5)_{F}$ the fields $S^{i j}$ break up as $S_{5} \oplus S_{0}$ transforming as $\mathbf{5}$ and $\mathbf{1}$ respectively. At the enhanced symmetry point, the $\lambda S M$ term in the superpotential yields

$$
W=\lambda M_{5} S_{5} \pm 2 \lambda \Lambda_{2}^{2} S_{0}
$$


The first term mixes the $\widehat{M}_{5}$ and $S_{5}$ moduli which are present for $\lambda=0$. The resulting Dirac states receive a small mass $m=\lambda \Lambda_{2}$. The second term is linear in the singlet component $S_{0}$. Supersymmetry is therefore broken by the $F$ component of $S_{0}$ with two degenerate vacua of energy $V \sim\left|\lambda^{2} \Lambda_{2}^{4}\right|$.

For $\lambda S^{i j} \gg \Lambda_{2}$, the fields $Q_{i}$ are heavy and can be integrated out by enforcing the $M_{i j}$ equations of motion. Treating $\lambda$ as a small parameter and restricting to the $M_{i j}$ quantum moduli space, the superpotential (2.2) in the $\lambda S^{i j} \gg \Lambda_{2}$ limit becomes

$$
W= \pm 2 \lambda \Lambda_{2}^{2} \sqrt{\operatorname{Pf} S}
$$

Because $\operatorname{Pf}(\partial W / \partial S)=\lambda^{2} \Lambda_{2}^{4} \neq 0$, supersymmetry is broken. In terms of the $S^{i j}$ components $S_{0}$ and $S_{5}$ introduced above, $\sqrt{\operatorname{Pf} S}=\sqrt{S_{0}^{2}-S_{5}^{2}} \approx S_{0}$ for $S_{0}^{2} \gg S_{5}^{2}$. The superpotential (2.5) amounts to a linear term for $S_{0}$ and gives $S_{5}$ a mass of order $m \sim \lambda \Lambda_{2}^{2} / S_{0}$. In this limit supersymmetry is again broken by the $F$ component of $S_{0}$ with two vacua of energy $V \sim\left|\lambda^{2} \Lambda_{2}^{4}\right|$.

The quantum modification of the moduli space, which breaks supersymmetry, can be given another interpretation in the $\lambda S^{i j} \gg \Lambda_{2}$ limit. In this limit, the quarks $Q$ are massive and can be integrated out as described above. The low energy theory then consists of the singlets $S^{i j}$ along with pure $S U(2)$ Yang-Mills theory with a scale $\Lambda_{L}$ related to that of the high energy theory by the matching condition $\Lambda_{L}^{6}=\lambda^{2}(\operatorname{Pf} S) \Lambda_{2}^{4}$. Gaugino condensation in the pure $S U(2)$ theory results in a superpotential $W_{L}= \pm 2 \Lambda_{L}^{3}$, giving precisely the superpotential (2.5). So supersymmetry breaking is described in terms of a quantum deformation of the classical moduli space for $\lambda S^{i j} \ll \Lambda_{2}$ while, for $\lambda S^{i j} \gg \Lambda_{2}$, it is possible to describe it in terms of gaugino condensation.

Note that the description of supersymmetry breaking in terms of gaugino condensation is entirely at the renormalizable level. This is in contrast to the usual discussion of supersymmetry breaking by gaugino condensation (within supergravity for example) which requires a non-renormalizable, moduli dependent gauge kinetic function. In addition, the usual discussion requires another mass scale to define a nontrivial curvature on the Kahler manifold of the modulus (or equivalently a non-linear gauge kinetic function) in order to stabilize the gaugino condensate against the Dine-Seiberg instability [8]. Without this, the theory is driven to weak coupling and supersymmetry is restored. Here, however, no additional mass scale is required to obtain stable supersymmetry breaking by gaugino condensation. 
This model has a pseudo-flat direction corresponding to the $S_{0}$ component of $S^{i j}$ along which $V \sim\left|\lambda^{2} \Lambda_{2}^{4}\right|$. This direction would be exactly flat if the Kahler potential for $S$ were precisely canonical. Quantum contributions to the Kahler potential $K=S_{0}^{\dagger} S_{0} f(t=$ $\left.\left|\Lambda_{2}\right|^{2} / S_{0}^{\dagger} S_{0}\right)$ can lift this degeneracy, giving the scalar potential $V(t)=4\left|\lambda^{2} \Lambda_{2}^{4}\right|\left(f-t f^{\prime}+\right.$ $\left.t^{2} f^{\prime \prime}\right)^{-1}$. The dominant quantum correction to the potential comes from integrating out the lightest states which, in the limit $\lambda S_{0} \gg \Lambda_{2}$, are the fields $S_{5}$ with mass $m \sim \lambda \Lambda_{2}^{2} / S_{0} \ll$ $\Lambda_{2}$. However, because these fields receive a supersymmetric mass to lowest order, this contribution to the vacuum energy vanishes at this order. The Kahler potential for $S_{0}$ is then smooth as $S_{0} \rightarrow \infty$ and $f(0)=1$. For finite $S_{0}$ there are corrections to the Kahler potential. Unfortunately, because the strongly coupled excited spectrum is incalculable, the precise function $f(t)$ can not be obtained. The natural scales for the minima along this direction are either $S_{0}=0, \mathcal{O}\left(\Lambda_{2}\right)$, or $\infty$. If the minimum does lie at $S^{i j}=0$, the $U(1)_{R}$ symmetry remains unbroken; otherwise, there is an $R$-axion in the massless spectrum. As emphasized in [9, [0] and also seen in [6], this illustrates that a spontaneously broken $U(1)_{R}$ symmetry is a sufficient but not necessary condition for supersymmetry breaking. If the minima are at $S_{0} \rightarrow \infty$, it may appear formally that the theory does not have a stable ground state. This is of course irrelevant to the question of supersymmetry breaking since the potential approaches a non-zero constant as $S_{0} \rightarrow \infty$.

The existence of this pseudo-flat direction may seem to contradict the common lore that models of dynamical supersymmetry breaking can not have flat directions. In contrast to flat directions along which a gauge group is Higgsed and becomes weaker, here the matter fields become more massive and the theory becomes more strongly coupled, leading to a vacuum energy which does not vanish even infinitely far along the pseudo-flat direction.

The scheme of obtaining supersymmetry breaking as the result of the quantum deformation of a moduli space by coupling singlets (or fields with weak gauge charges) to meson bilinears is easily extended to other gauge groups, such as $S P\left(N_{c}\right)$ with $N_{f}=N_{c}+1$ flavors of fundamental quarks [11]. Another generalization is to groups in which higher order invariants are required to parameterize the quantum moduli space, such as $S U\left(N_{c}\right)$ with $N_{f}=N_{c}$ flavors [3]. In this case, singlets must couple to each invariant which appears in the quantum constraint; otherwise, supersymmetry would be restored on some subspace. For example, in $S U\left(N_{c}\right)$ with $N_{c}$ flavors of fundamental quarks $Q_{i}$ and $\bar{Q}_{j}$, two singlets, $S$ and $\bar{S}$, may be coupled to the baryon invariants $B=Q^{N_{c}}$ and $\bar{B}=\bar{Q}^{N_{c}}$ as $W=\lambda_{1} \bar{S} B+\lambda_{2} S \bar{B}$. This coupling may be enforced, for example, by gauging baryon number. For $\lambda_{i} \neq 0$ this 
coupling lifts the $B \bar{B} \neq 0$ branch of the moduli space. However, the quantum constraint $\operatorname{det} M-B \bar{B}=\Lambda^{2 N_{c}}$ [3], where $M_{i j}=Q_{i} \bar{Q}_{j}$, is still satisfied on a moduli space parameterized by the $M_{i j}$ subject to $\operatorname{det} M=\Lambda^{2 N_{c}}$, with $S=\bar{S}=B=\bar{B}=0$. Coupling additional fields to all the $M_{i j}$ would completely lift the moduli space of supersymmetric vacua and lead to supersymmetry breaking.

\section{The $S U(3) \times S U(2)$ Model}

Perhaps the best known model of dynamical supersymmetry breaking is the $S U(3) \times$ $S U(2)$ model of Affleck, Dine, and Seiberg [2]. The usual discussion of supersymmetry breaking in this model has implicitly focused on the limit where the $S U(3)$ dynamics dominates and supersymmetry breaking is associated with a dynamically generated superpotential. In this section, we show that, in a limit where the $S U(2)$ dynamics dominates, supersymmetry breaking is associated with a quantum-deformed moduli space constraint.

The matter content of the model is

$$
\begin{array}{cc} 
& S U(3) \times S U(2) \\
P & (3,2) \\
L & (1,2) \\
\bar{U} & (\overline{3}, 1) \\
\bar{D} & (\overline{3}, 1) .
\end{array}
$$

This is just the one generation supersymmetric standard model without hypercharge, the positron, or Higgs bosons. Classically, this model has a moduli space parameterized by three invariants: $Z=P^{2} \overline{U D}, X_{1}=P L \bar{D}$, and $X_{2}=P L \bar{U}$. There is another gauge invariant, $Y=P^{3} L$, which vanishes classically by Bose statistics of the underlying fields. The gauge group is completely broken for generic vacua on the classical moduli space; the above invariants are the fields which are left massless after the Higgs mechanism. At tree level there is a single renormalizable coupling which can be added to the superpotential,

$$
W_{\text {tree }}=\lambda X_{1}
$$

This superpotential leaves invariant non-anomalous accidental $U(1)_{R}$ and $U(1)$ flavor symmetries, and completely lifts the classical moduli space. Classically, there is a supersymmetric ground state at the origin, with the gauge symmetries unbroken. 
Non-perturbative gauge dynamics generate an additional term in the effective superpotential; the exact effective superpotential is fixed by holomorphy, symmetries, and an instanton calculation to be

$$
W=\frac{\Lambda_{3}^{7}}{Z}+\mathcal{A}\left(Y-\Lambda_{2}^{4}\right)+\lambda X_{1}
$$

where $\mathcal{A}$ is a Lagrange multiplier field. The first term is generated by an instanton in the broken $S U(3)$; it is just the dynamical superpotential which would arise over the classical moduli space for $\Lambda_{2}=0$. The second term enforces the quantum deformed constraint $Y=P^{3} L=\Lambda_{2}^{4}$. This constraint can be seen in the limit $\Lambda_{2} \gg \Lambda_{3}$. In this limit the $S U(3)$ is weakly gauged at the scale $\Lambda_{2}$. The $S U(2)$ theory therefore has two flavors with a quantum deformed constraint [3]. Assuming the full Kahler potential is positive definite and nonsingular, (3.3) lifts the classical ground state $Z=X_{i}=0$. In the ground state of the quantum theory, both the $U(1)_{R}$ and supersymmetry are spontaneously broken [2,9, 90 .

The description of supersymmetry breaking depends on the relative importance of the dynamically generated $S U(3)$ superpotential and the $S U(2)$ deformation of the moduli space. In the limit $\Lambda_{3} \gg \Lambda_{2}$ the $S U(2)$ is weakly gauged at the scale $\Lambda_{3}$. The theory can then be analyzed by perturbing about the classical moduli space, $Y=0$, ignoring the second term in (3.3). For $\lambda \ll 1$ the vacuum expectation values of the fields in the ground state are close to the classical moduli space, and large compared to both $\Lambda_{2}$ and $\Lambda_{3}$. Both $S U(3)$ and $S U(2)$ are then Higgsed at a high scale and thus weakly coupled. In this weak coupling limit the Kahler potential $K$ may be approximated by the classical Kahler potential $K_{c l}$ for the elementary fields projected on the classical moduli space [2]. The vacuum expectation values of the fields and ground state energy can then be computed numerically in this limit [2,12]. Parametrically, for $\lambda \ll 1$, the field expectation values and vacuum energy scale as $\phi \sim \Lambda_{3} / \lambda^{1 / 7}$ and $V \sim\left|\lambda^{2}\left(\Lambda_{3} / \lambda^{1 / 7}\right)^{4}\right|=\left|\lambda^{10 / 7} \Lambda_{3}^{4}\right|$. In order for this approximation to be valid, the quantum deformation of the moduli space must be unimportant at the scale of the expectation values, which requires $\Lambda_{3} \gg \lambda^{1 / 7} \Lambda_{2}$. Notice that in this limit the $S U(2)$ acts a spectator in the non-perturbative dynamics which break supersymmetry. It restricts certain couplings which would otherwise be allowed in the superpotential, and it's classical gauge potential lifts certain directions in field space.

For $\Lambda_{2} \gg \Lambda_{3}$ the $S U(3)$ is weakly gauged at the scale $\Lambda_{2}$ and the quantum modification of the moduli space arising from the second term in (3.3) is important. For $\lambda \ll 1$, the relevant moduli space to perturb about is then the quantum one. Below the scale $\Lambda_{2}$, 
the theory can be described in terms of the light $S U(2)$ singlet fields $\widehat{q}=P L / \Lambda_{2}$ in the 3 of $S U(3)$ and $\widehat{\bar{q}}=P^{2} / \Lambda_{2}, \bar{U}$, and $\bar{D}$ all in the $\overline{\mathbf{3}}$ of $S U(3)$, subject to the quantum constraint $\widehat{q} \bar{q}=\Lambda_{2}^{2}$. The components of $\widehat{q}$ and $\widehat{\bar{q}}$ which preserve the constraint are just the components $\widehat{M}_{5}$ discussed in the previous section. On the quantum moduli space the $S U(3)$ is generically completely broken. At the point $\bar{U}=\bar{D}=0$ and $\widehat{q}=\widehat{\bar{q}}=\Lambda_{2}$ there is an unbroken $S U(2)^{\prime} \subset S U(3)$. Unlike the classical moduli space, there is no point at which the $S U(3)$ is restored. 1 For $\lambda \neq 0$, the classical supersymmetric ground state at which the full gauge symmetry is unbroken is therefore lifted by the quantum deformation of the moduli space. It follows that if the Kahler potential is positive definite and non-singular, supersymmetry is spontaneously broken by the quantum deformation of the $S U(2)$ moduli space.

Supersymmetry breaking by the quantum deformation of the moduli space can be illustrated by expanding about the enhanced symmetry point with an unbroken $S U(2)^{\prime} \subset$ $S U(3)$. At this point, the components of $\widehat{q}$ and $\widehat{\bar{q}}$ which satisfy the constraint are eaten by the Higgs mechanism. The low energy theory is $S U(2)^{\prime}$ with doublets $u$ and $d$ and singlets $S_{u}$ and $S_{d}$, all coming from the fields $\bar{U}$ and $\bar{D}$, with $X_{1}=\Lambda_{2}^{2} S_{u}$ and $X_{2}=\Lambda_{2}^{2} S_{d}$. The scale $\Lambda_{2^{\prime}}$ of the low energy theory is related to $\Lambda_{3}$ by the matching condition $\Lambda_{2^{\prime}}^{5}=\Lambda_{3}^{7} / \Lambda_{2}^{2}$ and the superpotential (3.3) yields the superpotential of the low energy theory,

$$
W=\frac{\Lambda_{2^{\prime}}^{5}}{u d}+\lambda \Lambda_{2}^{2} S_{d}
$$

The low energy theory also has $U(1)_{R}$ and $U(1)$ flavor symmetries. The first term in (3.4) is interpreted as coming from an instanton in the low energy $S U(2)^{\prime}$ with one flavor. This leads to a potential which pushes $u$ and $d$ away from the enhanced symmetry point. It follows from the $U(1)_{R}$ and $U(1)$ flavor symmetries that the superpotential in the low energy theory only depends on $S_{d}$ via the linear term in (3.4).

Since $S_{d}$ is a canonically normalized field in this limit, supersymmetry is broken by the $F$ component of $S_{d}$ with $V \sim\left|\lambda^{2} \Lambda_{2}^{4}\right|$. The low energy theory (3.4) appears to have a runaway direction labeled by $u d$, and pseudo-flat directions labeled by $S_{u}$ and $S_{d}$. In the effective theory, corrections to the Kahler potential from the strong $S U(2)$ dynamics lift these directions. In addition, for sufficiently large expectation values, the theory returns to a classical regime, in which the potential coming from the $D$ and $F$ terms rises in all

1 Note that the fields $\widehat{q}, \widehat{\bar{q}}, \bar{U}$, and $\bar{D}$ do not form an anomaly free representation of $S U(3)$. This is not inconsistent since $S U(3)$ is never restored on the quantum moduli space. 
directions in field space. The natural scale for the expectation value along $u d$ is therefore $\mathcal{O}\left(\Lambda_{2}\right)$, and for $S_{u}$ and $S_{d}$ either 0 or $\mathcal{O}\left(\Lambda_{2}\right)$. With these expectation values, in the limit $\lambda \Lambda_{2}^{2} \gg \Lambda_{2^{\prime}}^{5} / \Lambda_{2}^{3}$, which is equivalent to $\lambda^{1 / 7} \Lambda_{2} \gg \Lambda_{3}$, the $S U(2)^{\prime}$ dynamical superpotential in the low energy theory gives an insignificant contribution to the vacuum energy, and does not plays a role in the supersymmetry breaking. In this limit, supersymmetry breaking is associated with the quantum deformation of the $S U(2)$ moduli space, with the $S U(3)$ acting as a spectator. Unfortunately, because some of the fields are $\mathcal{O}\left(\Lambda_{2}\right)$, the Kahler potential receives large incalculable corrections, and a quantitative solution of the ground state of the effective theory in terms of the $u, d, S_{u}$ and $S_{d}$ is not possible.

\section{4. $S U(N) \times S P(M)$ Generalizations}

Many chiral models with two gauge groups exhibit the behavior illustrated in the previous section. In one limit, supersymmetry is broken by a dynamically generated superpotential in one gauge group while, in another limit, it is broken by the quantum deformation of a moduli space by another gauge group. The simplest such generalizations of the $S U(3) \times S U(2)$ model are given by theories with gauge group and matter content

$$
\begin{array}{ccc} 
& S U(N) \times S P\left(\frac{1}{2}(N-1)\right) \\
P & (N, N-1) & \\
L & (1, N-1) & \\
\bar{Q}_{i} & (\bar{N}, 1) & i=1, \ldots, N-1,
\end{array}
$$

with $N$ odd. These theories have a classical moduli space of vacua with the gauge group generically completely broken and light moduli $X_{i}=P L \bar{Q}_{i}$ and $Z_{i j}=P^{2} \bar{Q}_{i} \bar{Q}_{j}=-Z_{j i}$. In addition, there is another gauge invariant, $Y=P^{N} L$, which vanishes as a classical constraint. The classical moduli space degeneracy is lifted by the tree level superpotential

$$
W_{\text {tree }}=\lambda X_{1}+\sum_{i, j>2}^{N-1} \gamma^{i j} Z_{i j}
$$

where $\gamma^{i j}=-\gamma^{j i}$ has rank $N-3$. This superpotential leaves invariant some global flavor symmetries and a non-anomalous $U(1)_{R}$ symmetry. The form of the superpotential (4.2) could be enforced by weakly gauging certain subgroups of the flavor symmetries. As discussed in [13], the non-renormalizable terms in (4.2) are required for $N>3$ to completely lift the classical moduli space. All the $X_{i}$ and $Z_{1 j}$ are lifted by the first term 
in (4.2) while the remaining $Z_{i j}$ are lifted by the non-renormalizable terms. Classically, there is a supersymmetric ground state at the origin.

The superpotential in the quantum theory is

$$
W=\frac{\Lambda_{S U}^{2 N+1}}{\operatorname{Pf} Z}+\mathcal{A}\left(Y-\Lambda_{S P}^{N+1}\right)+W_{\text {tree }},
$$

where $\mathcal{A}$ is a Lagrange multiplier field. The first term arises from an instanton in the broken $S U(N)$ and the second enforces the quantum deformed constraint $Y=P^{N} L=\Lambda_{S P}^{N+1}$ arising from the $S P(M)$ dynamics [11].

The non-renormalizable terms in (4.2) introduce an additional scale beyond the dynamically generated scales. In order for (4.1) to have a weakly coupled regime below the scale of the non-renormalizable operators, we assume $\gamma^{i j} \ll \Lambda_{S U}^{-1}$ and $\Lambda_{S P}^{-1}$. The $Z_{i j}$ $i, j \geq 2$ moduli are lifted only by non-renormalizable terms and therefore have a much smaller classical potential than the other moduli in this limit. As a consequence, the quantum mechanical ground state develops large expectation values along these directions. For $\lambda \ll 1$ the full theory can then be analyzed in terms of the resulting effective theory. With $Z_{1 j}=0$, the expectation values $Z_{i j}$ have maximal rank $N-3$. With this maximal rank, the gauge group is classically broken to $S U(3) \times S U(2)$ with matter content given by (3.1) and the $\frac{1}{2}(N-2)(N-3)$ singlets $Z_{i j}, i, j \geq 2$. The $2(N-3)$ fields coming from the components of $\bar{Q}_{1}$ and $L$ which lie along the broken generators pair up and gain a large Dirac mass from the renormalizable term in (4.2). Along these directions the theory therefore reduces to the $S U(3) \times S U(2)$ model, with matter fields and a superpotential corresponding precisely to the theory of [2] and the previous section, along with the light singlets $Z_{i, j}, i, j \geq 2$, with the superpotential term in (4.2). The scales of the low energy $S U(3) \times S U(2)$ theory are related to those of the original theory by matching conditions $\widehat{\Lambda}_{3}^{7}=\Lambda_{S U}^{2 N+1} /\left(\operatorname{Pf}^{\prime} Z\right)$, and $\widehat{\Lambda}_{2}^{4}=\Lambda_{S P}^{N+1} / \sqrt{\operatorname{Pf}^{\prime} Z}$, evaluated at the scale $\left(\operatorname{Pf}^{\prime} Z\right)^{1 /(2 N-6)}$, where $\mathrm{Pf}^{\prime}$ is over the sub-matrix of $Z_{i j}$ with non-vanishing rank $N-3$. The dynamically generated superpotential (4.3) agrees with (3.3) upon using these matching relations, rescaling the Lagrange multiplier field as $\mathcal{A}^{\prime}=\mathcal{A} \sqrt{\mathrm{Pf}^{\prime} Z}$, and using $\operatorname{Pf} Z=P^{2} \overline{U D}\left(\operatorname{Pf}^{\prime} Z\right)$ and $Y=P^{N} L=\sqrt{\operatorname{Pf}^{\prime} Z} P^{3} L$, where $\bar{D}$ and $\bar{U}$ denote the components of $\bar{Q}_{1}$ and a linear combination of the $\bar{Q}_{i} i \geq 2$ which lie in the directions of the $S U(3)$ generators.

As in the previous section, in the limit $\lambda \ll 1$ and $\lambda^{1 / 7} \widehat{\Lambda}_{2} \ll \widehat{\Lambda}_{3}$, supersymmetry breaking in the effective theory is due to an instanton in the broken $S U(3)$ and the elementary fields of the $S U(3) \times S U(2)$ theory have $K \approx K_{c l}$. In order to obtain the dependence 
of the ground state expectation values and vacuum energy on the dynamical scales and couplings, we note that in this limit the superpotential is parametrically of the form

$$
W \sim \frac{\Lambda_{S U}^{2 N+1}}{A^{4} B^{2 N-6}}+\lambda A^{3}+\gamma B^{4}
$$

where $A$ are the fields (3.1) of the effective $S U(3) \times S U(2)$ theory while $B^{4}$ corresponds to the components of $Z_{i j}$ which gain large expectation values. Assuming $K \approx K_{c l}=$ $A^{\dagger} A+B^{\dagger} B$, the potential is then of the form

$$
V \sim\left|\frac{\Lambda_{S U}^{2 N+1}}{A^{5} B^{2 N-6}}+\lambda A^{2}\right|^{2}+\left|\frac{\Lambda_{S U}^{2 N+1}}{A^{4} B^{2 N-5}}+\gamma B^{3}\right|^{2},
$$

where the first term comes from $\partial_{A} W$ and the second from $\partial_{B} W$. The expectation values in the effective $S U(3) \times S U(2)$ theory are determined by a balance within the $\partial_{A} W$ term between the $S U(3)$ instanton and the renormalizable superpotential term, which gives the scaling $A \sim \widehat{\Lambda}_{3} / \lambda^{1 / 7} \sim\left(\lambda^{-1} \Lambda_{S U}^{2 N+1} B^{6-2 N}\right)^{1 / 7}$. This leads to a potential for the $B$ fields of the form

$$
V \sim\left|\frac{\lambda^{5} \Lambda_{S U}^{4 N+2}}{B^{4 N-12}}\right|^{2 / 7}+\left|\left(\frac{\lambda^{4} \Lambda_{S U}^{3(2 N+1)}}{B^{6 N-11}}\right)^{1 / 7}+\gamma B^{3}\right|^{2}
$$

The first term is just the contribution to the potential from the effective $S U(3) \times S U(2)$ theory. The remaining terms come from the $B$ dependence of the $S U(3)$ instanton and the non-renormalizable superpotential. Notice that the non-perturbative terms lift the potential for small values of $B$, while the non-renormalizable terms lift the potential at large expectation values. The position of the ground state is determined by a balance between these two types of terms. One possibility is that the $B$ dependence of the $S U(3)$ instanton and the non-renormalizable terms balance, with an insignificant contribution from potential of the effective $S U(3) \times S U(2)$ theory. This occurs for $\left(\gamma \Lambda_{S U}\right)^{2 N+1} \gg \lambda^{2 N+2}$. In this limit the expectation values scale as $B \sim\left(\lambda^{4}\left(\gamma \Lambda_{S U}\right)^{-7}\right)^{1 /(6 N+10)} \Lambda_{S U}$, giving a vacuum energy $V \sim\left|\left(\lambda^{12}(\gamma \Lambda)^{6 N-11}\right)^{1 /(3 N+5)} \Lambda_{S U}^{4}\right|$. In order for the approximation $K=A^{\dagger} A+B^{\dagger} B$ to be valid, the ground state must be weakly coupled and thus it should be that $B \gg \Lambda_{S U}, \Lambda_{S P}$, which requires $\left(\gamma \Lambda_{S U}\right)^{7} \ll \lambda^{4}$ and $\left(\lambda^{4}\left(\gamma \Lambda_{S U}\right)^{-7}\right)^{1 /(6 N+10)} \gg \Lambda_{S P} / \Lambda_{S U}$. In addition, the quantum modification of the moduli space in the effective theory is unimportant for $\lambda^{1 / 7} \widehat{\Lambda}_{2} \ll \widehat{\Lambda}_{3}$, which is equivalent to $\left(\lambda^{-4}\left(\gamma \Lambda_{S U}\right)^{(N-3) /(N+1)}\right)^{1 /(6 N+10)} \gg \Lambda_{S P} / \Lambda_{S U}$. Another simple situation to consider is the opposite limit, $\left(\gamma \Lambda_{S U}\right)^{2 N+1} \ll \lambda^{2 N+2}$, where the ground state is determined by a balance between the $B$ dependent potential of the effective $S U(3) \times S U(2)$ theory and the non-renormalizable terms, with an insignificant contribution from the $B$ dependence of the $S U(3)$ instanton. In this case the 
expectation values scale as $B \sim\left(\lambda^{5}\left(\gamma \Lambda_{S U}\right)^{-7}\right)^{1 /(4 N+9)} \Lambda_{S U}$, giving a vacuum energy $V \sim\left|\left(\lambda^{15}\left(\gamma \Lambda_{S U}\right)^{4 N-12}\right)^{2 /(4 N+9)} \Lambda_{S U}^{4}\right|$. For a weakly coupled ground state, $B \gg \Lambda_{S U}, \Lambda_{S P}$, which requires $\left(\gamma \Lambda_{S U}\right)^{7} \ll \lambda^{5}$ and $\left(\lambda^{5}\left(\gamma \Lambda_{S U}\right)^{-7}\right)^{1 /(4 N+9)} \gg \Lambda_{S P} / \Lambda_{S U}$. Finally, the quantum modification of the moduli space in the effective theory is unimportant in this limit for $\left(\lambda^{-3}\left(\gamma \Lambda_{S U}\right)^{(N-3) /(N+1)}\right)^{1 /(4 N+9)} \gg \Lambda_{S P} / \Lambda_{S U}$.

In the limit $\lambda^{1 / 7} \widehat{\Lambda}_{2} \gg \widehat{\Lambda}_{3}$, supersymmetry breaking in the effective theory is due to the quantum deformation of the $S U(2)$ moduli space. The potential in the effective theory in this limit is $V \sim\left|\lambda^{2} \widehat{\Lambda}_{2}^{4}\right|$, giving a potential for the $B$ of the form

$$
V \sim\left|\frac{\lambda^{2} \Lambda_{S P}^{N+1}}{B^{N-3}}\right|+\left|\gamma B^{3}\right|^{2}
$$

A balance between these terms gives $B \sim\left(\lambda\left(\gamma \Lambda_{S P}\right)^{-1}\right)^{2 /(N+3)} \Lambda_{S P}$, and vacuum energy $V \sim\left|\left(\lambda^{6}\left(\gamma \Lambda_{S P}\right)^{N-3}\right)^{2 /(N+3)} \Lambda_{S P}^{4}\right|$. In order for the ground state to be weakly coupled, $B \gg \Lambda_{S P}, \Lambda_{S U}$, which requires $\left(\lambda^{-1}\left(\gamma \Lambda_{S P}\right)\right)^{2 /(N+3)} \ll 1, \Lambda_{S U} / \Lambda_{S P}$.

\section{Quantum Removal of Classical Flat Directions}

Most models of supersymmetry breaking are constructed to have a $U(1)_{R}$ symmetry, and, as a result of $D$ and $F$ term constraints, no classical flat directions. The classical supersymmetric ground state with unbroken $U(1)_{R}$ is then at the origin of field space. If a non-perturbative dynamical superpotential lifts the origin, the $U(1)_{R}$ is spontaneously broken, and it follows that supersymmetry is broken [2,9, 10]. The examples of the previous two sections break supersymmetry in this manner in the limit that the $S U(N)$ dynamics dominates. Although the two conditions of having a $U(1)_{R}$ symmetry and no classical flat directions are generically sufficient for dynamical supersymmetry breaking, they are not necessary [9,10].

In the present section we show that models which have a classical flat direction can also break supersymmetry. This is counter to the usual intuition that quantum dynamics can not lift entire flat directions but can, at best, lead to a runaway potential with a supersymmetric ground state at infinity. This more familiar situation is bound to be the case when the entire gauge group is broken by the scalar expectation values, leading to gauge dynamics with a strength which tends to zero along the flat direction. However, it is possible for quantum dynamics to lift an entire flat direction, without leading to a runaway vacuum, if part of the gauge group is not broken along the flat direction. 
Models which illustrate the situation discussed above are based on

$$
\begin{array}{ccc} 
& S U(N) \times S P\left(\frac{1}{2}(N+1)\right) \\
P & (N, N+1) & \\
L & (1, N+1) & \\
\bar{Q}_{i} & (\bar{N}, 1) & i=1, \ldots, N+1
\end{array}
$$

with $N$ odd. There is a classical moduli space of vacua with the gauge group generically completely broken. The classical moduli are $X_{i}$ and $Z_{i j}$, as in the previous section, $\bar{B}^{i}=$ $\epsilon^{i j_{1} \ldots j_{N}} \bar{Q}_{j_{1}} \cdots \bar{Q}_{j_{N}}$, and $Y=P^{N} L$ (with $S U(N)$ indices contracted with an epsilon tensor) subject to the classical relations $Y \bar{B}^{i}=\left[X Z^{(N-1) / 2}\right]^{i}$ (antisymmetrizing over the flavor indices of $X$ and $Z$ ) and $\operatorname{Pf} Z=0$. These light fields correspond to the matter fields which are left massless after the Higgs mechanism. In addition, there are gauge invariant fields $H_{i}=P^{N+1} \bar{Q}_{i}$, which classically satisfy constraints $H_{i}=0$.

The tree level superpotential

$$
W_{\text {tree }}=\lambda X_{1}+\sum_{i, j>2}^{N+1} \gamma^{i j} Z_{i j},
$$

leaves invariant some global flavor symmetries and a non-anomalous $U(1)_{R}$ symmetry. All the $X_{i}, Y$, and $Z_{1 j}$ are lifted by the tree level term, $Z_{i j} i, j \geq 2$ are lifted by the non-renormalizable terms, but the $\bar{B}^{i}$ are left undetermined by (5.2). Classically there is moduli space of supersymmetric ground states given by $X_{i}=Y=Z_{i j}=0$ and $\bar{B}^{i}$ arbitrary. These theories only satisfy one of the two conditions mentioned above: there is a $U(1)_{R}$ symmetry but there are flat directions which are unlifted classically. As shown below, in the quantum theory the tree level superpotential (5.2) actually does lift all the flat directions and supersymmetry is broken. This is possible because the $S P(M)$ gauge group is unbroken along the entire classically unlifted flat direction.

The quantum theory is described by the above light fields with superpotential

$$
W=\frac{1}{\Lambda_{S U}^{2 N-1}}(\bar{B} H-\operatorname{Pf} Z)+\mathcal{A}\left(Y \operatorname{Pf} Z-H X Z^{(N-1) / 2}+\Lambda_{S U}^{2 N-1} \Lambda_{S P}^{N+4}\right)+W_{\text {tree }},
$$

where $\mathcal{A}$ is a Lagrange multiplier field. This form of the exact superpotential may be obtained, much as in the examples of [14], by considering the limit $\Lambda_{S U} \gg \Lambda_{S P}$. In this limit, the $S P(M)$ is weakly gauged at the scale $\Lambda_{S U}$. The $S U(N)$ theory therefore has $N_{f}=N+1$ flavors of fundamentals and confines into a theory of "mesons" $M_{i}=$ $P \bar{Q}_{i}$ which transform as $S P(M)$ fundamentals (as always, we display the flavor index, 
suppressing the $S P(M)$ gauge index), "baryons" $B=P^{N}$ which transform as an $S P(M)$ fundamental, and "antibaryons" $\bar{B}^{i}$ which are $S P(M)$ singlets. It follows from [3] that these confined fields have a superpotential corresponding to the first term in (5.3). Below the scale $\Lambda_{S U}$, the $S P(M)$ has $N_{f}=M+1$ flavors $(2 M+2=N+3$ matter fields $)$ in the fundamental representation, corresponding to the fields $\widehat{M}_{i}=M_{i} / \Lambda_{S U}$, the field $\widehat{B}=B / \Lambda_{S U}^{N-1}$, and the field $L$. Notice that, because of the extra confined degrees of freedom which becomes massless at the origin, the $S P(M)$ theory below the scale $\Lambda_{S U}$ has one more flavor than the underlying high energy theory. The scale $\widehat{\Lambda}_{S P}$ of the low energy theory with one extra flavor is related to $\Lambda_{S P}$ by the matching relation $\Lambda_{S P}^{N+4}=\Lambda_{S U} \widehat{\Lambda}_{S P}^{N+3}$ at the confining scale $\Lambda_{S U}$. $S P(M)$ with $N_{f}=M+1$ flavors has a quantum deformed moduli space [11], which is enforced by the second term in (5.3). The scale $\Lambda_{S U}$ appears in the quantum deformed constraint because factors of $\Lambda_{S U}$ in $\widehat{\Lambda}_{S P}, \widehat{M}_{i}$, and $\widehat{B}$, reflecting the matching conditions and the fact that some of the canonically normalized $S P(M)$ flavors are composites associated with the $S U(N)$ confinement.

We now consider the limit in which the $S P(M)$ dynamics dominate, $\Lambda_{S U} \ll \Lambda_{S P}$. The first term in (5.3), arising from the $S U(N)$ confinement, becomes large in this limit. Imposing equations of motion which receive contributions from this term amounts to enforcing classical constraints on the $S U(N)$ moduli space. In particular, $\bar{B}^{i}$ and $H_{i}$ get a large mass in this limit and hence can be integrated out by imposing their equations of motion, leading to the classical constraint $H_{i}=0$ along with $\bar{B}^{i}=\Lambda_{S U}^{2 N-1} \mathcal{A}\left(X Z^{(N-1) / 2}\right)^{i}$. In addition, the Pfaffian of the $Z_{i j}$ equations of motion, and $H_{i}=0$, imply $\mathcal{A}=1 /\left(\Lambda_{S U}^{2 N+1} Y\right)$ in this limit. Together with the $H_{i}$ equations of motion this yields the classical constraint $Y \bar{B}^{i}=\left[X Z^{(N-1) / 2}\right]^{i}$, and the superpotential (5.3) becomes

$$
W=\frac{\Lambda_{S P}^{N+4}}{Y}+\mathcal{A}^{\prime}\left(Y \operatorname{Pf} Z+\Lambda_{S U}^{2 N-1} \Lambda_{S P}^{N+4}\right)+W_{\text {tree }},
$$

where $\mathcal{A}^{\prime}$ is a different Lagrange multiplier field. The first term is generated by an instanton in the broken $S P(M)$. The second term enforces the quantum deformed constraint $Y \operatorname{Pf} Z=-\Lambda_{S U}^{2 N-1} \Lambda_{S P}^{N+4}$ associated with the $S P(M)$ dynamics. In order for the $S P(M)$ instanton superpotential to be finite requires $Y \neq 0$. For $\Lambda_{S U}=0$, the constraint enforced by the $\mathcal{A}^{\prime}$ equation of motion therefore yields the classical constraint $\operatorname{Pf} Z=0$ associated with the classical $S P(M)$ moduli space, l $^{2}$ and the superpotential becomes

$$
W=\frac{\Lambda_{S P}^{N+4}}{Y}+W_{\text {tree }}
$$

2 The classical constraint $\operatorname{Pf} Z=0$ can also be obtained for $\Lambda_{S U} \neq 0$ in the theory with $W_{\text {tree }}=$ 
The dynamically generated instanton superpotential is just that which would arise over the classical moduli space for $\Lambda_{S U}=0$.

The lifting of the classical flat directions $\bar{B}^{i}$ and supersymmetry breaking can be seen in the $\Lambda_{S U} \ll \Lambda_{S P}$ limit in the effective theory (5.5) subject to the classical constraints among the gauge invariants. In this limit the $S P(M)$ instanton potential in (5.5) leads to $Y \neq 0$ in the quantum theory. For $Y \neq 0$, the constraint $Y \bar{B}=X Z^{(N-1) / 2}$ can be used to relate the $\bar{B}^{i}$ flat directions to $X_{i}$ and $Z_{i j}$ flat directions. Because $W_{\text {tree }}$ lifts the $X_{i}$ and $Z_{i j}$ flat directions, it eliminates all flat directions in the quantum theory. Since $Y$ is also lifted by $W_{\text {tree }}$, there is a stable ground state with the $U(1)_{R}$ symmetry is spontaneously broken. It follows that supersymmetry is broken. In this limit the classical flat directions are lifted by the tree level superpotential in the presence of the dynamically generated $S P(M)$ superpotential. We conclude that it is possible for theories with classical flat directions to dynamically break supersymmetry with a stable vacuum.

\section{Resolving the Origin}

In order to analyze the issue of supersymmetry breaking, it is crucial that all of the relevant light fields entering in the low energy effective theory be properly identified. Neglecting some light fields on a moduli space can lead to the mistaken conclusion that a theory breaks supersymmetry while, upon proper inclusion of all relevant fields, it is seen that the theory actually has a supersymmetric vacuum. As a simple example, consider a Wess-Zumino theory with chiral superfields $L$ and $H$ with canonical Kahler potential and superpotential $W=L H^{2}$. The field $L$ is massless and can have arbitrary expectation value. For $L \neq 0, H$ is massive and can be integrated out, yielding the low energy superpotential $W=0$. Adding a superpotential $W=\mu^{2} L$ to the low energy theory with $H$ integrated out appears to break supersymmetry, $\partial_{L} W \neq 0$. But in the theory with $H$ included, there is a supersymmetric ground state at $L=0$, and $H^{2}=-\mu^{2}$. In order to realize that supersymmetry is unbroken, a low energy observer must know that, in addition to the light field $L$, there is another field $H$ which becomes massless at $L=0.3$

0 by integrating out $Y$ and enforcing the $\mathcal{A}^{\prime}$ equation of motion. This gives the superpotential $W=-\operatorname{Pf} Z / \Lambda_{S U}^{2 N-1}$. The $Z_{i j}$ equations of motion yield the classical constraint $\operatorname{Pf} Z=0$, and the $\mathcal{A}^{\prime}$ equation of motion implies $Y \rightarrow \infty$.

3 Exactly this situation occurs for $N=2$ Yang-Mills theory broken explicitly to $N=1$ YangMills theory by giving a mass to the adjoint $\Phi$. There $L \sim \operatorname{Tr} \Phi^{2}$ and $H$ corresponds to the 
Models which break supersymmetry by a dynamically generated superpotential become strongly coupled for small field values since the dynamical superpotential becomes singular at the origin. A quantitative analysis does not seem possible in this region. One might worry that such models could develop another branch in this strong coupling region along which supersymmetry is unbroken, as in the toy example given above. One way to gain confidence that there are no such strong coupling subtleties, which could lead to supersymmetric vacua near the origin, is to consider theories with additional, massive, vector-like matter integrated in. Additional matter makes the gauge dynamics more weakly coupled, leading to a more weakly coupled description of the supersymmetry breaking. The dynamics of the original theory is recovered in the limit that the mass of the vector matter is taken much larger than all dynamical scales in the theory. Extensions of known supersymmetry breaking models with additional vector-like matter have been considered previously in 16,17,18.

Depending on how many massive vector-like matter fields are added, it is possible to realize supersymmetry breaking in the low energy theory via a variety of mechanisms. In the present context it is most useful to add enough vector-like matter so that all the gauge groups become confining. The region of field space which was strongly coupled in the original model is then weakly coupled in the theory with additional vector matter. Extra confined degrees of freedom do in fact become light near the origin. However, since the gauge groups are confined, if supersymmetry is broken it must be realized as a tree level effect for the confined fields. The low energy theory with additional vector like matter then amounts to an O'Raifeartaigh-type model, in which supersymmetry breaking can be easily verified.

As an example, consider the $S U(3) \times S U(2)$ theory with matter content

$$
\begin{array}{ccc} 
& S U(3) \times S U(2) & \\
P & (3,2) & \\
L_{a} & (1,2) & a=1, \ldots, 3 \\
Q & (3,1) & i=1, \ldots, 3 .
\end{array}
$$

This is just the $S U(3) \times S U(2)$ model of [2] and section 3 with an extra, vector-like, $S U(3)$ flavor and an extra, vector-like, $S U(2)$ flavor. The classical moduli space is parameterized by the invariants $M=P^{2} Q, X_{a, i}=P L_{a} \bar{Q}_{i}, Y_{a}=P^{3} L_{a}, M_{i}=Q \bar{Q}_{i}, B=P^{3} L_{a} L_{b} L_{c} \epsilon^{a b c}$, monopoles which are massless at strong coupling [15]. 
$B^{a}=Q\left(P L_{b}\right)\left(P L_{c}\right) \epsilon^{a b c}, \bar{B}=\bar{Q}_{i} \bar{Q}_{j} \bar{Q}_{k} \epsilon^{i j k}, \bar{B}^{i}=\epsilon^{i j k} P^{2} \bar{Q}_{j} \bar{Q}_{k}$, and $V^{a}=\frac{1}{2} \epsilon^{a b c} L_{b} L_{c}$ subject to the classical relations $M \bar{B}=-M_{i} \bar{B}^{i}, Y_{a} \bar{B}=-X_{a, i} \bar{B}^{i}, M B=-Y_{a} B^{a}, M_{i} B=$ $-X_{a, i} B^{a}, B \bar{B}=\operatorname{det} X, B^{a} \bar{B}=-\frac{1}{2} M_{i} X_{b, j} X_{c, k} \epsilon^{a b c} \epsilon^{i j k}, B \bar{B}^{i}=-\frac{1}{2} Y_{a} X_{b, j} X_{c, k} \epsilon^{a b c} \epsilon^{i j k}$, and $B^{a} \bar{B}^{i}=\frac{1}{2} M X_{b, j} X_{c, k} \epsilon^{a b c} \epsilon^{i j k}-Y_{b} M_{j} X_{c, k} \epsilon^{a b c} \epsilon^{i j k}$. The tree level superpotential

$$
W_{\text {tree }}=\lambda X_{1,1}+m_{Q} M_{3}+m_{L} V^{1}
$$

leaves invariant non-anomalous $U(1)_{R}$ and $U(1)$ flavor symmetries, and completely lifts the moduli space. Classically there is a supersymmetric ground state at the origin.

The quantum theory is described by the above light fields with superpotential

$$
\begin{aligned}
W= & -\frac{1}{\Lambda_{2}^{3}}\left(B-Y_{a} V^{a}\right)+\frac{1}{\Lambda_{2}^{3} \Lambda_{3}^{6}}\left(M B \bar{B}+Y_{a} B^{a} \bar{B}+M_{i} B \bar{B}^{i}+X_{a, i} B^{a} \bar{B}^{i}\right. \\
& \left.-M \operatorname{det} X+\frac{1}{2} Y_{a} M_{i} X_{b, j} X_{c, k} \epsilon^{a b c} \epsilon^{i j k}\right)+W_{\text {tree }} .
\end{aligned}
$$

This form of the exact superpotential may be obtained by considering the limit $\Lambda_{2} \gg$ $\Lambda_{3} \gg m_{Q}, m_{L}$. In this limit, the $S U(3)$ is weakly coupled at the scale $\Lambda_{2}$. The $S U(2)$ theory therefore has 3 flavors of fundamentals and confines into a theory of "mesons" $V^{a}$ which are $S U(3)$ singlets, $P L / \Lambda_{2}$ in the $\mathbf{3}$ of $S U(3)$, and $P^{2} / \Lambda_{2}$ in the $\overline{\mathbf{3}}$ of $S U(3)$. The superpotential for these confined fields corresponds the the first term in (6.3) [3]. Because of the extra confined $S U(2)$ mesons which become massless at the origin, the $S U(3)$ theory below the scale $\Lambda_{2}$ has one more flavor than the underlying high energy theory. The scale $\widehat{\Lambda}_{3}$ of the low energy theory with one extra flavor is related to $\Lambda_{3}$ by the matching condition $\Lambda_{3}^{6}=\Lambda_{2} \widehat{\Lambda}_{3}^{5}$ at the confining scale $\Lambda_{2}$. The $S U(3)$ theory with 4 flavors confines [3] into a theory of "mesons" $\widehat{M}=M /\left(\Lambda_{2} \Lambda_{3}\right), \widehat{X}_{a, i}=X_{a, i} /\left(\Lambda_{2} \Lambda_{3}\right), \widehat{Y}_{a}=Y_{a} /\left(\Lambda_{2}^{2} \Lambda_{3}\right), \widehat{M}_{i}=M_{i} / \Lambda_{3}$, and "baryons" $\widehat{B}=B /\left(\Lambda_{2}^{3} \Lambda_{3}^{2}\right), \widehat{B}^{a}=B^{a} /\left(\Lambda_{2}^{2} \Lambda_{3}^{2}\right), \widehat{\bar{B}}=\bar{B} / \Lambda_{3}^{2}$, and $\widehat{\bar{B}}^{i}=\bar{B}^{i} /\left(\Lambda_{2} \Lambda_{3}^{2}\right)$. These fields, along with the $S U(2)$ meson $\widehat{V}^{a}=V^{a} / \Lambda_{2}$, make up the canonically normalized confined fields which are light near the origin. The $S U(3)$ confining superpotential is given by the second term in $(6.3)$. As in the previous section, the scale $\Lambda_{2}$ arises in this term because of the matching relation and normalization factors of $\Lambda_{2}$ coming from the $S U(3)$ flavors which are composites associated with the $S U(2)$ confinement.

For $m_{Q}, m_{L} \ll \Lambda_{3}, \Lambda_{2}$, and $\lambda \ll 1$ the expectation values in the ground state are much smaller than the dynamical scales, and the superpotential (6.3) is the relevant one to consider. In this limit the theory just amounts to an O'Raifeartaigh-type model for the confined degrees of freedom, with tree level couplings (6.3). Supersymmetry is broken if 
all the auxiliary $F$ components can not simultaneously vanish. Some of the $F$ terms are

$$
\begin{aligned}
\left(X^{a, i}\right)_{F}^{*} & =\frac{1}{\Lambda_{3}^{6} \Lambda_{2}^{3}}\left(B^{a} \bar{B}^{i}-\frac{1}{2} M X_{b, j} X_{c, k} \epsilon^{a b c} \epsilon^{i j k}+Y_{b} M_{j} X_{c, k} \epsilon^{a b c} \epsilon^{i j k}\right)-\lambda \delta^{a, 1} \delta^{i, 1} \\
\left(M^{i}\right)_{F}^{*} & =\frac{1}{\Lambda_{3}^{6} \Lambda_{2}^{3}}\left(B \bar{B}^{i}+\frac{1}{2} Y_{a} X_{b, j} X_{c, k} \epsilon^{i j k} \epsilon^{a b c}\right)+m_{Q} \delta^{i, 3} \\
\left(V_{a}\right)_{F}^{*} & =\frac{1}{\Lambda_{2}^{3}} Y_{a}+m_{L} \delta_{a, 1} \\
(\bar{B})_{F}^{*} & =\frac{1}{\Lambda_{2}^{3} \Lambda_{3}^{6}}\left(M B+Y_{a} B^{a}\right) .
\end{aligned}
$$

Note first that $Y_{a}\left(X^{a, 3}\right)_{F}^{*}+M\left(M^{3}\right)_{F}^{*}-\bar{B}^{3}(\bar{B})_{F}^{*}=M m_{Q}$. Vanishing $F$ terms therefore require $M=0$ for $m_{Q} \neq 0$. It then follows that $B^{a}\left(V_{a}\right)_{F}^{*}-\Lambda_{3}^{6}(\bar{B})_{F}^{*}=m_{L} B^{1}$ and, therefore, $B^{1}=0$ for $m_{L} \neq 0$. In addition, $\left(V_{a}\right)_{F}^{*}=0$ implies $Y_{a}=0, a \neq 1$. Finally, it follows from $M=B^{1}=Y_{a}=0, a \neq 1$, that $\left(X^{1,1}\right)_{F}^{*}=-\lambda$. So all the $F$ terms in (6.4) can not vanish for non-zero $m_{Q}, m_{L}$, and $\lambda$, and supersymmetry is therefore broken. In any theory of confined fields which breaks supersymmetry, there must be at least one term in the superpotential which is linear in a confined field. Otherwise, all fields can sit at the origin with supersymmetry unbroken.

For $m_{Q}, m_{L} \gg \Lambda_{2}, \Lambda_{3}$ the additional vector-like matter can be integrated out, yielding the $S U(3) \times S U(2)$ model (3.1) with superpotential (3.3). This can be verified explicitly in this limit by using the matching relations $\widehat{\Lambda}_{3}^{\prime 7}=m_{Q} \Lambda_{3}^{6}$ and $\widehat{\Lambda}_{2}^{\prime 4}=m_{L} \Lambda_{2}^{3}$, and integrating out all the fields in (6.3) but $Z=\bar{B}^{3}, X_{1}=X_{1,1}, X_{2}=X_{1,2}$, and $Y=Y_{1}$, which are just the standard $S U(3) \times S U(2)$ invariants. We therefore see explicitly that supersymmetry is broken in both the large and small mass limit. Any additional branches at strong coupling in the original $S U(3) \times S U(2)$ model along which supersymmetry could be restored would presumably survive in the theory with additional vector matter, and appear at the origin as additional massless fields in the weakly coupled, small mass, limit. This is not the case, and supersymmetry is indeed broken at tree level in this weakly coupled limit. This gives confidence that there are no such strong coupling subtleties in the $S U(3) \times S U(2)$ model. Extension to other models which break supersymmetry by dynamically generated superpotentials are straightforward.

\section{Conclusions}

The quantum deformation of a classical moduli space can lead to supersymmetry breaking. This occurs if the quantum deformed constraint associated with the moduli 
space is inconsistent with a stationary superpotential. The vacuum energy and auxiliary components for some fields then do not vanish. It may at first sight seem surprising that supersymmetry can be broken even though the dynamical superpotential on the classical moduli space exactly vanishes. In fact, the absence of a dynamical superpotential over the classical moduli space has often been given as a "proof" that supersymmetry could not be broken in such theories. However, it is possible that the tree level auxiliary components only vanish at points on the classical moduli space which are removed by the quantum deformation, thus leading to supersymmetry breaking. In some instances it is possible to analyze models which break supersymmetry by this mechanism by expanding about enhanced symmetry points on the quantum moduli space. The Kahler potential for the non-perturbative fields which become light at these points is approximately canonical, and often allows a quantitative description of the relevant degrees of freedom in the ground state.

The $S U(2)$ model discussed in section 2 has a number of interesting features aside from demonstrating supersymmetry breaking by the quantum modification of a moduli space. It is the first example of a renormalizable model in which singlet fields participate directly in the supersymmetry breaking. This can have phenomenological applications to both hidden sector and gauge mediated supersymmetry breaking. Hidden sector singlets which participate directly in supersymmetry breaking are required in renormalizable hidden sector models in order to obtain dimension three soft terms of order the weak scale [19]. Without this, gauginos are much lighter than the weak scale in such scenarios [19,20]. Likewise, in gauge mediated supersymmetry breaking, singlets with both scalar and $F$ components of order $S \sim \sqrt{F_{S}}$ are required in many schemes [13, 21]. The singlets couple through Yukawa couplings, $h S Q_{m} \bar{Q}_{m}$, to vector quarks $Q_{m}$ and $\bar{Q}_{m}$ which transform under the standard model gauge group. The vector quarks act as messengers of supersymmetry breaking; integrating them out generates soft and explicit supersymmetry breaking in the low energy theory. In order to obtain $S \sim \sqrt{F_{S}}$ previous schemes have invoked a secondary messenger sector with nonzero $D$ terms to couple the supersymmetry breaking sector with the singlets [13,21]. In the $S U(2)$ model given here, however, it is possible that the singlets have a minimum with $S \sim \sqrt{F_{S}} \sim \Lambda_{2}$ for $\lambda \sim 1$. Again, the location of the minimum along the pseudo-flat direction depends on the quantum corrections to the Kahler potential which, unfortunately, can not be computed quantitatively. It is a natural possibility, though, for a minimum to exist at $\mathcal{O}\left(\Lambda_{2}\right)$. Note, however, that simply adding 
the messenger quark coupling $h S Q_{m} \bar{Q}_{m}$ to the model of section 2 would introduce a supersymmetric ground state with $F_{S}=0$ and $Q_{m} \overline{Q_{m}} \neq 0$. The desired minimum with $F_{S} \neq 0$ and $Q_{m} \bar{Q}_{m}=0$ could only be a local minimum. In any case, this model demonstrates that it is possible in principle to consider schemes without the secondary messenger sector.

Another interesting feature of the $S U(2)$ model is that it is non-chiral with respect to the gauge group, an aspect stressed in [7] and very recently also in [22]. This appears to conflict with the Witten index [23] argument that $\operatorname{Tr}(-1)^{F} \neq 0$ in non-chiral models and supersymmetry is, therefore, unbroken. It is, in fact, possible to have $\operatorname{Tr}(-1)^{F}=0$ in nonchiral models when, as in the $S U(2)$ model, there is a pseudo-flat direction which extends to infinity. This allows the index to change under small deformations of the model since vacua can move in or out from infinity along this direction. When the pseudo-flat direction degeneracy is lifted by quantum effects as discussed in section 2 , the vacuum has $\operatorname{Tr}(-1)^{F}=$ 0 and supersymmetry is broken. If, however, the singlets $S^{i j}$ are given a mass $\epsilon$ by adding a term $\epsilon$ Pf $S$ to the superpotential, the index becomes $\operatorname{Tr}(-1)^{F}=2$ as expected for $S U(2)$ [23], and there are two supersymmetric vacua. To understand this change in the index, consider the low energy theory along the pseudo-flat direction $S_{0}$ with $W= \pm 2 \lambda \Lambda_{2}^{2} S_{0}+\epsilon S_{0}^{2}$. For $\epsilon=0$, the vacuum energy along the entire $S_{0}$ direction is $V \sim\left|\lambda^{2} \Lambda_{2}^{4}\right|$. However, for $\epsilon \neq 0$, there are two supersymmetric ground states at $S_{0}= \pm \lambda \Lambda_{2}^{2} / \epsilon$. For $\epsilon \rightarrow 0$ these ground states are sent to $\infty$ along the flat direction. In this way, $\operatorname{Tr}(-1)^{F}$ is discontinuous at $\epsilon=0$; the theory with $\epsilon=0$ (enforced by discrete symmetries or by gauging certain subgroups of the flavor symmetry) breaks supersymmetry whereas that with $\epsilon \neq 0$ does not. This pathologic behavior of the index occurs only when there is a pseudo-flat direction in the quantum theory and does not occur for the chiral models discussed in this paper. Such behavior is expected for any non-chiral model of dynamical supersymmetry breaking. For example, pure gaugino condensation with a moduli dependent gauge kinetic function exhibits the same behavior.

The models of section 5 provide examples in which a flat direction remains in the classical theory, but is lifted, with supersymmetry broken, in the quantum theory. The standard list of "requirements" for stable dynamical supersymmetry breaking was assumed to include: chiral matter, no classical flat directions which extend to infinity, a dynamically generated superpotential, and a spontaneously broken $U(1)_{R}$ symmetry. The difficulty in finding models which simultaneously satisfy all these requirements lead to the belief that dynamical supersymmetry breaking was difficult and perhaps not generic. As shown in 
Refs. [9,10 a $U(1)_{R}$ symmetry is in fact not necessary. We now see that the other "requirements" are also not necessary. It would be interesting to find a single model which satisfies none of the standard "requirements" but, nevertheless, breaks supersymmetry.

Models which break supersymmetry by a dynamically generated superpotential become strongly coupled near the origin. As discussed in section 6, integrating in extra vector-like matter makes the gauge dynamics more weakly coupled and allows the region of strong coupling to be resolved. New vacua for which supersymmetry might be restored do not appear in the theory with vector-like matter integrated in. It is in fact unlikely that such additional massless states could appear as functions of the known moduli without ruining the 't Hooft anomaly matchings. Although this is of course not a proof, it does provide confidence that there are no such branches at strong coupling in the original theory without vector matter.

Finally, we note that although the discussion throughout has been in terms of rigid supersymmetry in which supersymmetry breaking implies a non-zero vacuum energy, generalization of any of the mechanisms discussed here to supergravity is straightforward.

\section{Acknowledgments}

We would like to thank M. Dine, A. Nelson, R. Rattazzi, and N. Seiberg for useful discussions. The work of K.I. was supported by NSF grant PHY-9513835 and the W.M. Keck Foundation. The work of S.T. was supported by the Department of Energy under contract DE-AC03-76SF00515 and the National Science Foundation under grant PHY94-07194. We would also like to thank the Aspen Center for Physics and Institute for Theoretical Physics where this work was partially completed. 


\section{References}

[1] I. Affleck, M. Dine, and N. Seiberg, Phys. Lett. B 137 (1984) 187; Phys. Rev. Lett. 52 (1984) 1677.

[2] I. Affleck, M. Dine, and N. Seiberg, Nucl. Phys. B 241 (1984) 493; Nucl. Phys. B 256 (1985) 557.

[3] N. Seiberg, hep-th/9402044, Phys. Rev. D 49 (1994) 6857.

[4] N. Seiberg, hep-th/9411149, Nucl. Phys. B 435 (1995) 129.

[5] K. Intriligator and N. Seiberg, hep-th/9509066

[6] K. Intriligator, N. Seiberg, and S. Shenker, hep-ph/9410203, Phys. Lett. B 342 (1995) 152.

[7] K. Intriligator and S. Thomas, talk presented at Unification: From the Weak Scale to Planck Scale, Institute for Theoretical Physics, Santa Barbara, CA, Oct. 23, 1995.

[8] M. Dine and N. Seiberg, Phys. Lett. B 162 (299) 1985

[9] N. Seiberg, hep-ph/9309335, Phys. Lett. B 318 (1993) 469.

[10] A. Nelson and N. Seiberg, hep-ph/9309299, Nucl. Phys. B 426 (1994) 46.

[11] K. Intriligator and P. Pouliot, hep-th/9505006, Phys. Lett. B 353 (1995) 471.

[12] J. Bagger, L. Randall, and E. Poppitz, hep-ph/9405345, Nucl. Phys. B 426 (1994) 3.

[13] M. Dine, A. Nelson, Y. Nir, and Y. Shirman, hep-ph/9507378, Phys. Rev. D 53 (1996) 2658.

[14] K. Intriligator, R.G. Leigh and N. Seiberg, hep-th/9403198, Phys. Rev. D 50 (1994) 1092.

[15] N. Seiberg and E. Witten, hep-th/9407087, Nucl. Phys. B 426 (1994) 19.

[16] E. Poppitz and S. Trivedi, hep-th/9507169, Phys. Lett. B 365 (125) 1996.

[17] H. Murayama, hep-ph/9505082, Phys. Lett. B 355 (1995) 187.

[18] P. Pouliot and M.J. Strassler, RU-95-78, hep-th/9602031.

[19] M. Dine and D. Macintire, hep-ph/9205227, Phys. Rev. D 46 (2594) 1992; T. Banks, D. Kaplan, and A. Nelson, hep-ph/9308292, Phys. Rev. D 49 (1994) 779.

[20] G. Farrar, RU-95-25, hep-ph/9508291; J. Feng, N. Polonsky, and S. Thomas, hepph/9511324, Phys. Lett. B 370 (95) 1996.

[21] M. Dine and A. Nelson, hep-ph/9303230, Phys. Rev. D 48 (1993) 1277; M. Dine, A. Nelson, and Y. Shirman, hep-ph/9408384, Phys. Rev. D 51 (1995) 1362.

[22] K. Izawa and T. Yanagida, hep-th/9602180.

[23] E. Witten, Nucl. Phys. B 202 (1982) 253. 